\title{
Mechanical characterization of elastic membranes: Cell mechanics applications
}

\author{
Roberto Bernal, Chantal Tassius and Francisco Melo \\ Departamento de Física, Universidad de Santiago de Chile, \\ and Center for Advanced Interdisciplinary Research in Materials (CIMAT), \\ Av. Ecuador 3493, Casilla 307, Correo 2, Santiago de Chile. \\ Jean-Christophe Géminard \\ Laboratoire de Physique, Ecole Normale Supérieure de Lyon, \\ 46 Allée d'Italie, 69364 Lyon cedex 07, France.
}

\begin{abstract}
Wrinkled patterns produced by cells crawling onto elastic membranes provide a useful tool to test living cells' locomotion. The technique however requires a characterization of the intrinsicproperties and tension of the membrane as well as an accurate determination of the deformation induced by the cell. Here, we present a capillary-type technique to estimate the elastic modulus of such membranes, describe an indentation technique to measure the initial tension, and develop a simple interferometric method to measure the amplitude field of the wrinkled pattern produced, for instance, by the action of living cell.
\end{abstract}


Wrinkling in elastic films under stress is ubiquitous in nature [1-3]. Common systems exhibiting this phenomenon are our skin [4], textiles and more specifically thin coatings subjected to stresses mismatch [5,6]. Apart from a basic interest, wrinkled membranes have found unexpected applications recently. For instance, in modern technology, pre-wrinkled conductive films are proposed as stretchable electrical contacts required in large-area electronics [7]. In medicine, surgery has always involved cutting, thickening contraction and in many cases wrinkling of the skin. Potential applications of wrinkle mechanics to improve wound healing have been recently summarized in Ref. [4]. In turn, in cell biomechanics, wrinkled patterns produced by cells crawling onto elastic membranes have provided a useful tool to test living cells' locomotion. With this method, in which membranes are obtained by vulcanization of the free surface of polymeric fluids, the wrinkle length has been correlated to the force applied by the cell cytoskeleton [1,9]. In a recent article [10], we reported an experimental and theoretical study of wrinkles formed in an initially flat elastic membrane, locally subjected to an axi-symmetric tangential force. We show that wrinkles appear as a consequence of a supercritical instability : both the length and amplitude of the wrinkles increase as the imposed force is increased beyond a well defined critical value which is a function of the membrane tension. Beyond the instability threshold, the number of nucleated wrinkles is nearly independent of the applied force, varies very slowly with membrane tension, and is a linear function of the size of the object responsible for the wrinkling i.e., the typical dimension of the region (relative to the membrane thickness) in which the cell attaches to the substrate. By contrast, the wrinkle length and amplitude proved to be very sensitive to the membrane tension, both quantities decreasing as the tension is increased. Moreover, the length resulted a slowly varying function of applied force, which challenges the sensitivity of cellular force measurements based on the direct measurement of the length. We suggested thus that a considerable more sensitive measurement can be obtained if the force is correlated directly to the amplitude instead. However, such a program requires the development of simple techniques to carry out film characterization and wrinkle topography measurements. To overcome these difficulties, here we present a protocol to characterize elastic membranes used in cell locomotion measurements and we develop an interferometric method to account for the wrinkle amplitude.

The membranes are prepared by vulcanization, as described in Refs. [1, 9], of a polymeric fluid, either PMP (phenylmethyl polysiloxane, Dow Corning 710) or PDMS (poly dimethyl 
siloxane, Sylgard 184). First, a thin fluid film is formed by spin coating on a clean piece of silicium wafer $(\mathrm{Si}$ : B[111], P/E) : A thickness of about $20 \mu \mathrm{m}$ is achieved after spinning at $4500 \mathrm{rpm}$ during $2 \mathrm{~min}$. Then, an elastic thin membrane, a few nanometers in thickness, is produced at the free surface by the direct effect of a homogeneous flame from a Bunzer-type burner. In order to insure the final membrane homogeneity, the silicium sample is displaced at constant speed by means of a motorized mount, the contact time of the flame with the fluid surface being of about $1 \mathrm{~s}$.

The first relevant quantity necessary for the interpretation of the wrinkled pattern is the membrane thickness, $h$. However, in our experimental configuration, nondestructive measurements are difficult. For instance, optical methods do not provide enough contrast as the membrane floats on top of a fluid having similar refraction-index. Hence, we propose to make use of an atomic force microscope. However, such a technique necessitates to separate the membrane from the liquid, which we propose to achieve by transferring, by mechanical contact, onto a clean piece of silicium wafer (Fig. 1). The fluid, which may remain at the new created surface, is removed by spinning the whole. The cleaning process is improved by adding a small drop of an appropriate solvent (isopropilic ethanol). Immediately after spinning in order to avoid contamination, we measure the difference in height between two regions across the film edge by means of an atomic force microscope (AFM) in the contact mode (Fig. 1). The preparation protocol described above is found to lead to PMP (resp. PDMS) membranes having reproducible thicknesses $h=(18 \pm 1) \mathrm{nm}[$ resp. $h=(60 \pm 2) \mathrm{nm}]$.

The second relevant quantity is the $2 \mathrm{D}$ elastic modulus $B \equiv E h /\left(1-\sigma^{2}\right)$ associated with the stretching of the membrane (We denote $\sigma$ the Poisson ratio and $E$ the Young modulus): In principle, provided that the membrane is thick enough, $B$ can be determined from the force-displacement relation or Hertz law [8], $F(\delta)=\frac{4}{3} \frac{E}{1-\sigma^{2}} \sqrt{r} \delta^{\frac{3}{2}}=\frac{4}{3} B \frac{\sqrt{r}}{h} \delta^{\frac{3}{2}}$, observed during the indentation of the dry membrane lying on the solid substrate with the AFM tip (Fig. 1c, $r$ stands for the radius of curvature of the AFM tip). However, the relation holds true only when the indenter is pushed against an elastic half-space: In our experimental configuration, the membranes are so thin that the measurements are inaccurate and altered by finite size effects (the thickness $h$ compares to $r$ ). We propose thus the capillarity-type method sketched in Fig. 2 instead. By using the vulcanization protocol described above, an initially flat membrane is prepared at the open end of a tube (radius $r_{0}=1 \mathrm{~mm}$ ) filled with the polymeric fluid (Fig. 2a). We then impose a pressure difference, $\Delta P$, across the membrane 
and, imaging the system from the side, we measure the resulting curvature $\mathcal{C}$ of the free surface. In the case of a liquid interface, the Laplace law would apply and $\Delta P=2 \Gamma \mathcal{C}$ with $\mathcal{C}$ the curvature of the interface cross-section and $\Gamma$ the surface tension (or free energy). In the case of an elastic membrane, the tension of the interface is not a thermodynamical quantity, which we shall denote $T_{0}$, in order to avoid confusion. For a membrane, the minimization of the elastic energy [11] leads to a non-linear relation between the pressure difference $\Delta P$ and the curvature $\mathcal{C}$,

$$
\Delta P \approx 2 T_{0} \mathcal{C}+2.66 B \frac{\arcsin (\mathcal{C} R)-\mathcal{C} R}{R},
$$

where, the elastic term, proportional to $B$, accounts for the increase in the tension resulting from the increase in the surface. Thus, the measurement of the non-linear term provides us with an estimate of the elastic modulus $B$ (Fig. 2b). We get $B=(240 \pm 10) \mathrm{mN} / \mathrm{m}$ $\left[T_{0}=(60.5 \pm 0.5) \mathrm{mN} / \mathrm{m}\right]$ for the PDMS membrane, and $B=(20 \pm 10) \mathrm{mN} / \mathrm{m}\left[T_{0}=(27 \pm 1)\right.$ $\mathrm{mN} / \mathrm{m}$ ] for the PMP membrane. We mention that we can estimate from the values of $B$ the Young modulus $E \simeq 3 \mathrm{MPa}$ (resp. $E \simeq 0.8 \mathrm{MPa}$ ) of the PDMS (resp. PMP) membranes.

The third relevant quantity is the tension $T_{0}$ of the membrane. As $T_{0}$ depends on the preparation of the system, the measurements from the capillary-type method are not applicable for the samples used in the biology experiment, and we propose here in situ measurements, instead. We choose to push vertically on the flat membrane with the suitable tip (Fig. 2c). In this case, the force-displacement relation $F(\delta)$ satisfies [11],

$$
F \approx \xi B^{1-\nu} T_{0}^{\nu} \delta
$$

where $\xi=1.143 \pm 0.002$ and $\nu=0.881 \pm 0.003$ have been obtained by numerically solving the elasticity problem. As an example, we made use of a microneedle to measure the force-displacement relation in the same experimental condition as in Fig. 2c for the PDMS membrane. The slope indicates $T_{0} \simeq 64 \mathrm{mN} / \mathrm{m}$, in fairly good agreement with the value $T_{0} \simeq 60.5 \mathrm{mN} / \mathrm{m}$ obtained from the pressure-curvature relation (Fig. $2 \mathrm{~b}$ ).

Finally, in the biology experiment, the geometry of the wrinkle-pattern can be characterized by using a Michelson type interferometer in which the sample acts as one of the mirrors. We made use of two objective lenses (Carl Zeiss CP Acromat, $10 \times / 0.25$ ), a dichroic cube of 25 mm side, a discharge Na-source, and a video camera (Nikon DXM 1200, 12 Megapixels). The sample consists of fibroblast-TYPE cells, cultured following the standard procedure 
described in [9], attached to a PMP membrane prepared as described above on a silicon wafer. An O-ring around the substrate and a cover glass form a narrow container, about $0.5 \mathrm{~mm}$ depth, for the culture medium and fibroblasts. By imposing a small tilt of the second mirror, one produces regularly-spaced parallel-fringes which are distorted when the membrane wrinkles (Fig. 3a). The contrast is increased by locating a drop of optical fluid on the second mirror of the interferometer and the wrinkles are better visualized when they are perpendicular to the fringes. The profile of each of the wrinkle, in a plane perpendicular to the sample plane, can be reconstructed from the local displacement of the fringes (Fig. 3b\&c). The vertical resolution achieved is about $25 \mathrm{~nm}$ whereas the spatial resolution is better than $5 \mu \mathrm{m}$.

In order to demonstrate the applicability of the method, let us now interpret, in the framework of our previous theoretical analysis of the problem [10], the wrinkle pattern described in Fig. 3, which results from the contraction of a single fibroblast. We expect

from the theory the wrinkle maximum-amplitude $A \simeq \sqrt{h r_{0}} \sqrt{3 \frac{\tau-T_{0}}{B}}$, where $\tau$ stands for the tension imposed by the cell in the adhesion region of typical size $r_{0}$. From the experimental $A$ and $r_{0}$ (Fig.3), we obtain $\tau \simeq 40 \mathrm{mN} / \mathrm{m}$ or forces, $\tau r_{0} \simeq 200 \mathrm{nN}$, which are equivalent to those reported on [9]. However, it should be noticed that the dynamical range for force measurement is limited. First, radial forces smaller than $T_{0}$ can not be detected and second for a force twice $T_{0}$ the cell requires to contract by $r_{0} / 3$ since $B / T_{0}$ is of the order of unity. As described in [9] this difficulty is overcome by tuning the membrane elastic properties by either increasing $B$ (thicker membranes) or by weakening the membrane by the use of $U V$ light. In conclusion, the protocol, which we proposed for the preparation and characterization of the membrane, provides us with the thickness $h \simeq 20 \mathrm{~nm}$, the tension $T_{0} \simeq 27 \mathrm{mN} / \mathrm{m}$ and the elastic modulus $B \simeq 20 \mathrm{mN} / \mathrm{m}$, which together with interferometry methods allows us to measure, in situ, the field amplitude of a wrinkled pattern produced by living cells on a silicone substrate. Our optical method, in addition, might prove useful for a variety of related applications, for instance, the study of the evolution of the cell body itself.

\section{Acknowledgments}

This work was supported by Conicyt-Chile under Fondap Program $N^{o} 11980002$. 


\section{BIBLIOGRAPHY}

[1] A. K. Harris, P. Wild and D. Stopak, Science, 208, 177, (1980).

[2] E. Cerda, K. Ravi-Chandar and L. Mahadevan, Nature, 419, 579, (2002); E. Cerda and L. Mahadevan, Phys. Rev. Lett., 90, 1, (2003).

[3] N. Sridhar, D. J. Srolovitz and Z. Suo, Applied Physics Letters, 75 N17, 2482, (2001).

[4] E. Cerda, J Biomech., 38 (8), 1598, (2005).

[5] See for instance, K. D. Hobart, F. J. Kub, M. Fatemi, M. E. Twigg, P. E. Thompson, T. S. Kuan, and C. K. Inoki, J. Electron. Mater., 29 (7), 897, (2000).

[6] K. D. Hobart, Special Issue on Alternative Substrate Technology - Foreword, J. Electron Mater. 30 (7), 797, (2001).

[7] S. P. Lacour, S. Wagner, Z. Y. Huang and Z. Suo. Applied Physics Letters 82 (15), 2404, (2003).

[8] L. D. Landau and E. M. Lifchitz, Theory of Elasticity (Pergamon, New York, 1959).

[9] K. Burton and D. L. Taylor, Nature 393, 150, (1998); K. Burton, J. H. Park and D. L. Taylor, Molecular Biology of the Cell, 10, 3745, (1999).

[10] J-Ch. Géminard, R. Bernal and F. Melo, Eur. Phys. J. E., 15, 117, (2004).

[11] R. Bernal, Ch. Tassius, J-Ch. Géminard and F. Melo, in preparation. 


\section{FIGURE CAPTIONS}

FIG. 1: In order to obtain a clean membrane deposited on a silicon wafer, we approach the free surface of the sample from the solid surface (a), which results in a piece of membrane underneath a thin fluid layer (b). The remaining fluid is removed by spinning at large velocity (c). The thickness $h$ [here $(17.8 \pm 0.2) \mathrm{nm}$ ] is measured at one edge of the membrane [image in the optical microscope (d)] which vertical profile (e) is obtained by using the AFM in the contact mode.

FIG. 2: (a) A pressure difference $\Delta P$ is imposed, by using the principle of communicating vessels, across an initially flat membrane prepared at the upper end of a capillary tube; (b) The elastic

modulus $B$ associated with the stretching of the membrane is obtained from the non-linearity in the relation between $\Delta P$ and the resulting curvature $\mathcal{C}$; (c) In the case of a membrane floating on a flat liquid layer, one can obtain in situ measurement of the tension $T_{0}$ by pushing vertically the free surface by means of a rounded tip; (d) The force $F$ is proportional to the displacement $\delta$, which is accounted by Eq. 2 .

FIG. 3: The wrinkle pattern results from the contraction of a single fibroblast which is moving freely on the elastic surface. Left panel; equal thickness fringe pattern modified by wrinkles. Right panel; amplitude of membrane deflection in gray scale. The wrinkles make an angle of about 8 degrees between them and the typical radius $r_{0}$ of the region in which they merge is of about 3 $\mu \mathrm{m}$. Lower panel; profile of the wrinkles: maximum vertical displacement along the wrinkles as a function of distance from the cell body. $W_{i}$ indexes the wrinkle $i$ on the top-right panel. The maximum amplitude $A$ is of about $0.3 \mu \mathrm{m}$ and the length $L$ about $150 \mu \mathrm{m}$. 
(a)

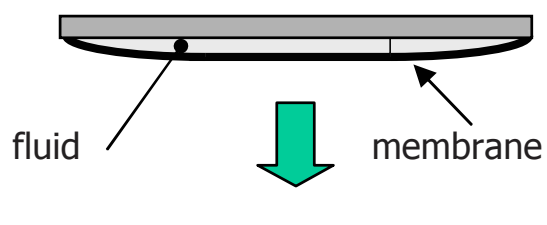

Si wafer (b)

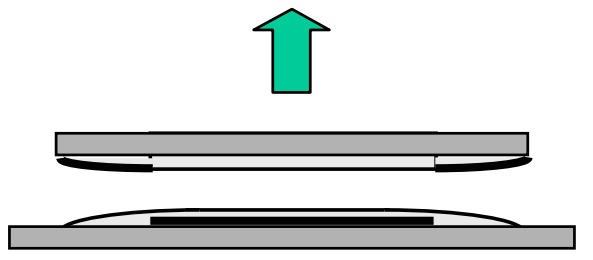

(c)

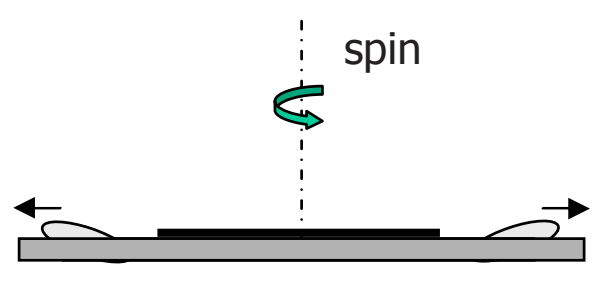

(d)

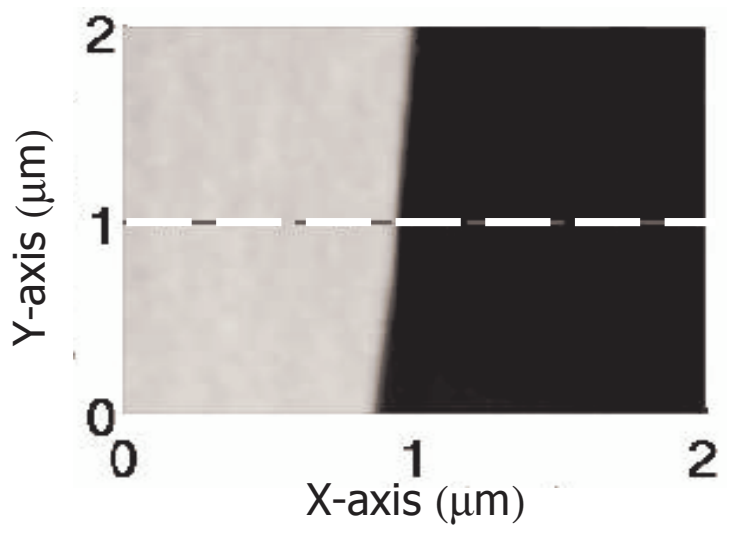

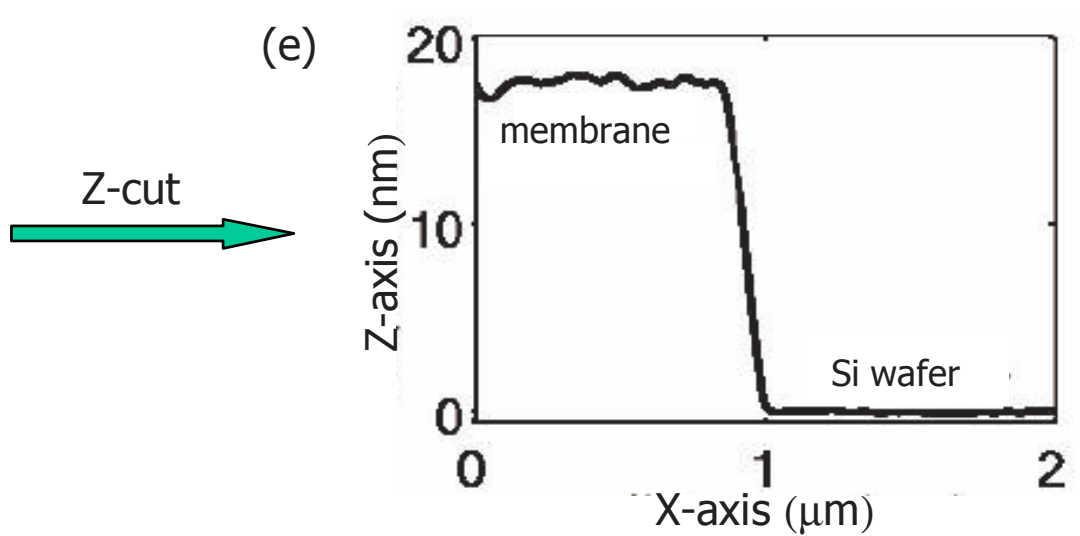


(a)

(c)

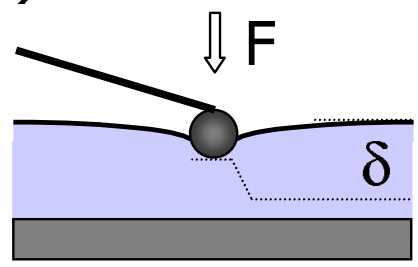

$\Delta \mathrm{P}$

$\longleftarrow 2 r_{0}$

C

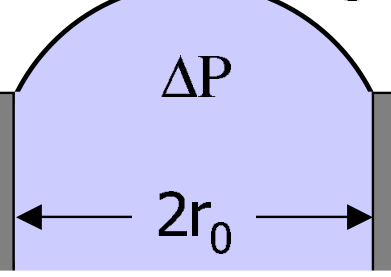

(b) 50

(d)

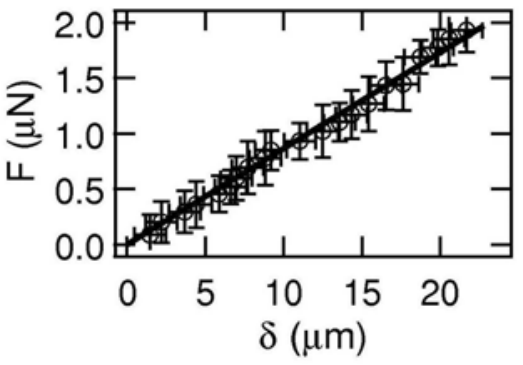


